\title{
Highly divergent neurovirulent vaccine-derived polioviruses of all three serotypes are recurrently detected in Finnish sewage
}

M Roivainen (merja.roivainen@thl.fi) ${ }^{1}$, Soile Blomqvist ${ }^{1}$, Haider al-Hello ${ }^{1}$, Anja Paananen ${ }^{1}$, Frances Delpeyroux ${ }^{2,3}$, M Kuusi $^{1}$,

T Hovi ${ }^{1}$

1. National Institute for Health and Welfare, Helsinki, Finland

2. Departement de Virologie, Biologie des Virus Entériques, Institut Pasteur, Paris, France

3. National Institute of Health and Medical Research, INSERM, U994, Paris, France

Citation style for this article:

Roivainen M, Blomqvist S, al-Hello H, Paananen A, Delpeyroux F, Kuusi M, Hovi T. Highly divergent neurovirulent vaccine-derived polioviruses of all three serotypes are recurrently detected in Finnish sewage. Euro Surveill. 2010;15(19): pii=19566. Available online: http://www.eurosurveillance.org/ViewArticle.

aspx?Articleld=19566

This article has been published on 13 May 2010

In Finland, surveillance of potential re-emergence of poliovirus transmission is mainly based on environmental surveillance, i.e. search for infectious poliovirus in sewage samples. Since December 2008, 21 genetically highly divergent, neurovirulent vaccinederived polioviruses (VDPV) have been isolated from sewage in Tampere, Finland. While the source of the VDPV is unknown, characteristics of the viruses resemble those of strains isolated from immunodeficient, persistently infected persons. No cases of suspected poliomyelitis have been reported in Finland since 1985 .

\section{Introduction}

Polioviruses are causative agents of acute paralytic disease, poliomyelitis (polio). Because of the work of the Global Polio Eradication Initiative and intensive polio immunisation programmes, wild polio virus is currently endemic only in four countries worldwide: Afghanistan, Pakistan, India, Nigeria. While the eradication of wild poliovirus gets closer, genetically drifted vaccine-derived polioviruses (VDPV) with regained neurovirulence, have become a new challenge for the eradication of polio [1-3]. In an individual vaccinated with oral poliovirus vaccine (OPV), the three vaccine strains (Sabin strains) infect the intestinal epithelium and replicate in the gut for a period ranging between several weeks and a couple of months. During this time, polioviruses undergo rapid genetic changes, which can result in the reversion of attenuating mutations. Very rarely, this may result in paralytic disease, the vaccine-associated paralytic poliomyelitis (VAPP), in vaccinees or in their immediate contacts. In suboptimally immunised populations the emergence of transmission of neurovirulent circulating vaccine-derived polioviruses (cVDPV) may occur. Several outbreaks caused by cVDPV have been documented [3-4]. In most cases, cVDPV have been detected soon after the onset of the first case of paralysis, but genetic analyses of isolated poliovirus strains have confirmed their silent circulation for at least one year before detecting the symptomatic case.

Since decades, polio surveillance in Finland has been carried out mainly through environmental surveillance. Between 1985, when a nationwide OPV campaign was launched to stop a polio epidemic which started in late 1984, and 2006 when an OPV-like poliovirus strain was isolated from sewage in Helsinki, no polioviruses were found in Finnish sewage. Since 2006, at least one Sabin-like poliovirus has been isolated from sewage almost every year. In Finland, only the inactivated poliovirus vaccine (IPV) has been used in vaccination campaigns, both before and after the 1985 OPV campaign. In spite of this Sabin-like poliovirus is occasionally detected in sewage. However, this is not surprising as a great number of people from OPV-using countries visit Finland every year. In this study we describe a more unexpected finding, namely the recurrent detection of neurovirulent and highly divergent VDPV strains of all three poliovirus serotypes, in Finnish sewage.

\section{Methods}

Sewage specimens were collected and analysed according to the recommendations made by the World Health Organization (WHO) [5-6]. Isolated poliovirus strains were characterised for serotype, intratype (ITD) and genetic properties as previously described [7].

\section{Results}

The first two highly divergent VDPV strains were isolated from a sewage specimen collected on 15 December 2008, in Tampere. The subsequent, intensified, weekly sampling revealed an additional 20 poliovirus strains (serotypes 1-3) in Tampere up to late March 2010 (Table).

Five of seven poliovirus-positive sewage samples contained more than one serotype and one specimen even contained several parallel strains from all three 
serotypes. A poliovirus type 1 strain isolated from a sample collected on 14 April 2009 was shown to be Sabin-like in ITD assays and was not characterised further. All other poliovirus strains gave aberrant results in ITD assays and were therefore subjected to partial genomic sequencing.

\section{Genetic relationships of vaccine- derived polioviruses}

The nucleotide sequences encoding the complete viral capsid protein 1 were determined from 21 Finnish poliovirus strains. The strains shared only $85.4-87.7 \%$ nucleotide and $92.7-95.7 \%$ amino acid identities with the parental Sabin strains of corresponding serotypes, indicating that poliovirus strains had evolved from OPV, but were highly divergent VDPV. The extent of sequence divergence suggests that the viruses have been replicated in humans for more than 10 years. When the viral capsid protein 1 sequences were compared with all sequences available in the GenBank and in the other laboratories of the Global Polio Laboratory Network, no close genetic relatives were found, indicating unique evolution pathways of these strains. The comparison with past and still existing wild poliovirus genotypes definitely excluded the wild-type origin of the strains (Figure).

Analysis of the reciprocal genetic relationships of VDPV showed that distinct strains of poliovirus type 1 and 3 , some isolated from separate sewage samples, were

\section{TABLE}

Strains of poliovirus isolated during environmental surveillance in Tampere, Finland, December 2008 to March 2010

\begin{tabular}{|c|c|c|}
\hline Specimen code & Collection date & Poliovirus serotype \\
\hline 2849 & 15.12 .2008 & $\mathrm{PV}_{1}$ \\
\hline 2849 & 15.12 .2008 & $\mathrm{PV}_{2}$ \\
\hline 2963 & $14 \cdot 4 \cdot 2009$ & $\mathrm{PV}_{1}$ \\
\hline 3001 & 15.6 .2009 & $\mathrm{PV}_{3}$ \\
\hline 3008 & 6.7 .2009 & $P V_{1}$ \\
\hline 3008 & 6.7 .2009 & $\mathrm{PV}_{1}$ \\
\hline 3008 & 6.7 .2009 & $\mathrm{PV} 1$ \\
\hline 3008 & 6.7 .2009 & $\mathrm{PV} 2$ \\
\hline 3008 & 6.7 .2009 & $\mathrm{PV} 2$ \\
\hline 3008 & 6.7 .2009 & $\mathrm{PV} 2$ \\
\hline 3008 & 6.7 .2009 & $\mathrm{PV}_{3}$ \\
\hline 3077 & $14 \cdot 9 \cdot 2009$ & $\mathrm{PV}_{1}$ \\
\hline 3077 & $14 \cdot 9.2009$ & $\mathrm{PV}_{1}$ \\
\hline 3077 & $14 \cdot 9 \cdot 2009$ & $\mathrm{PV}_{1}$ \\
\hline 3077 & 14.9 .2009 & $\mathrm{PV}_{3}$ \\
\hline 3077 & 14.9 .2009 & $\mathrm{PV}_{3}$ \\
\hline 3253 & 1.2 .2010 & $\mathrm{PV}_{1}$ \\
\hline 3253 & 1.2 .2010 & $\mathrm{PV}_{3}$ \\
\hline 3311 & 22.3 .2010 & $\mathrm{PV}_{1}$ \\
\hline 3311 & 22.3 .2010 & $\mathrm{PV}_{1}$ \\
\hline 3311 & 22.3 .2010 & $\mathrm{PV}_{2}$ \\
\hline 3311 & 22.3 .2010 & $\mathrm{PV}_{2}$ \\
\hline
\end{tabular}

closely related to each other, but none of the strains were completely identical (Figure). Six poliovirus type 2-VDPV strains segregated into two discrete genetic lineages and representatives of both lineages were isolated from the same sewage sample collected on 6 July 2009. Analyses of partial 3D polymerase coding sequences showed that all VDPV have the closest similarity to poliovirus type 1 Sabin strain in this genomic region indicating that type 2 and 3 VDPV had recombinant genomes. Intertypic recombination of the Sabin strains is a common phenomenon in recipients of OPV.

\section{Loss of attenuation in Finnish vaccine-derived polioviruses}

The representative VDPV strains of each of the three serotypes were tested for attenuation or neurovirulence in transgenic mice expressing the human poliovirus receptor (PVR-Tg 21 mice) [8]. PVR Tg-21 mice were inoculated intraperitoneally and/or intracerebrally with either VDPV isolates or Sabin strains. In contrast to Sabin strains, all tested VDPV induced paralysis or death in at least some of the inoculated animals. These results indicate that all tested VDPV have lost the attenuated phenotype that characterises the vaccine strains.

\section{Discussion and conclusion}

Highly divergent VDPV of all three serotypes were recurrently isolated from Tampere sewage. The molecular analysis of the viral capsid protein 1 coding regions revealed that all strains had originated from an OPV dose given more than 10 years ago.

The source of the VDPV remains unknown, but both epidemiological and genetic data suggest that they might be originally derived from (a) chronically infected still unidentified immunodeficient individual(s). It is well recognised that sometimes, when patients with hypogammaglobulinaemia are infected either by direct administration of OPV or as a consequence of contact with someone who received a dose of OPV, the virus excretion may continue for years or even for the rest of their lives [1-2,4]. Until now approximately 40 chronically poliovirus-infected individuals have been confirmed worldwide $[1,4,9]$. Two of them have prolonged infection with two poliovirus serotypes, while the others are excreting only one serotype $[4,9]$. While the VDPV strains of all three serotypes detected in Tampere most likely were originally established in one person, we cannot exclude the possibility that some of the viruses have spread to close contacts.

The identification of chronic excretors is of high importance, since patients with primary immunodeficiencies (i.e. having defects in antibody production) have increased risk of developing VAPP (estimated as a 3,000-fold risk) [10]. Since hypogammaglobulinemia has been reported to occur most often in Caucasians, the problem of chronic excretors of VDPV is highlighted in Europe and North America [1]. Furthermore, patients with hypogammaglobulinemia only have a 
longer life-expectancy in better resourced countries with a well developed health system. Persistent excretion of neurovirulent polioviruses poses a risk for transmission of the virus to susceptible contacts in the community, in spite of the lack of reports of paralytic cases among contacts of VDPV excreting immunodeficient individuals. While there is no medical treatment capable of interrupting poliovirus excretion, the risk of VAPP in an immunodeficient patient and his close contacts can be lowered by booster vaccinations with inactivated polio vaccine (IPV).

Although the loss of attenuation and regaining of neurovirulence were evident in all VDPV serotypes in transgenic mice, no poliovirus-induced paralyses have been found in Tampere or elsewhere in Finland since

\section{FIGURE}

A phylogenetic tree depicting the reciprocal genetic relationships of Finnish vaccine-derived poliovirus strains supplemented with sequences of wild-type polioviruses of each poliovirus serotype.

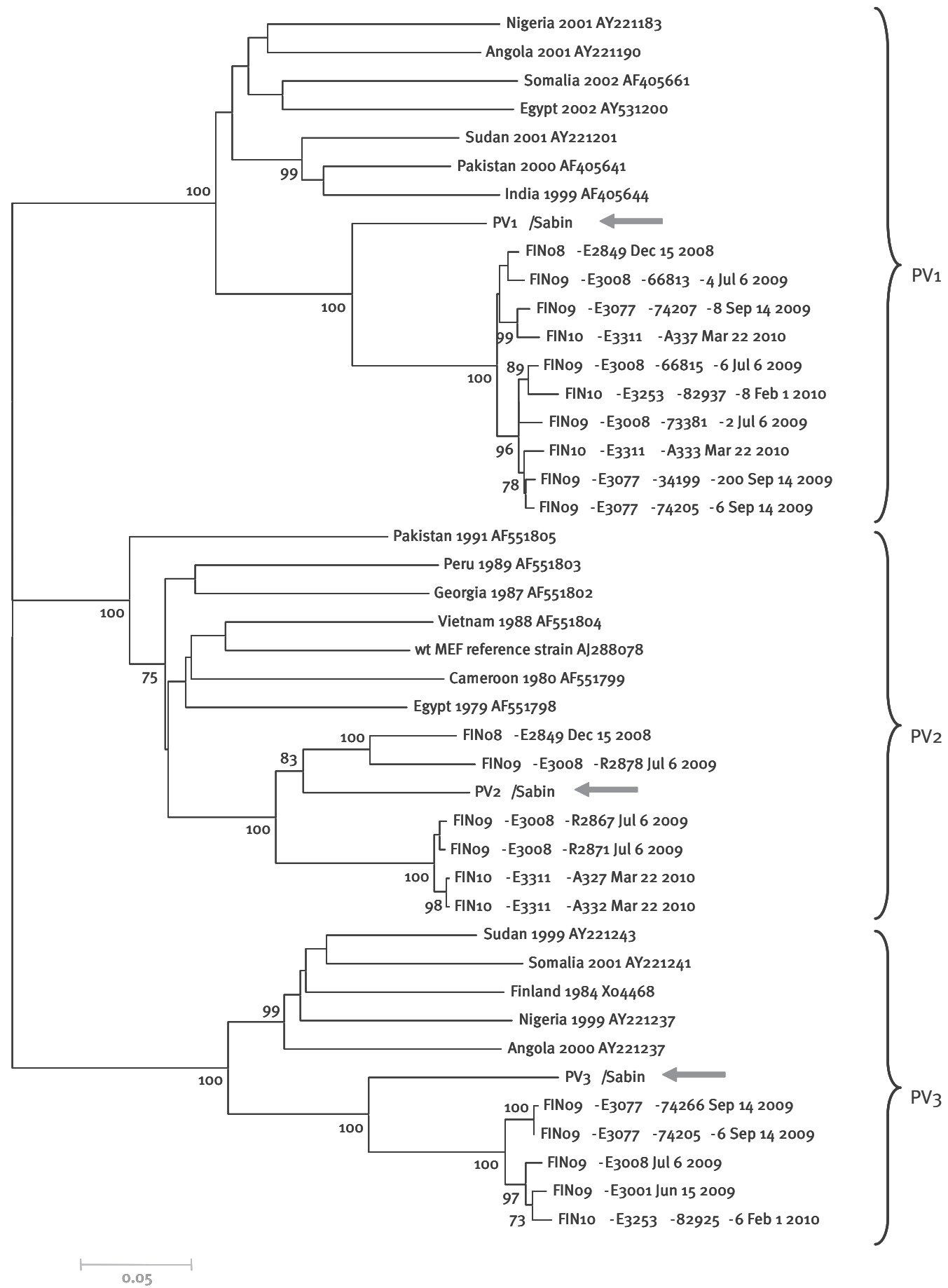

The tree was constructed from nucleotide sequences using neighbour-joining algorithm with Kimura 2-parameter substitution model. The consensus tree from 1000 replicate analyses is shown. The Finnish vaccine-derived poliovirus strains are in bold. The arrows indicate the parental OPV strains. The GenBank accession numbers and the country and year of isolation are shown for wild-type poliovirus strains. 
1985. The recurrent isolation of neurovirulent, highly divergent VDPV from Tampere sewage emphasises the importance of maintaining high polio vaccination coverage, but also highlights the usefulness and importance of environmental surveillance in poliovirus control.

\section{Acknowledgements}

We gratefully acknowledge the important contribution of Tampereen vesi for collecting sewage specimens from Tampere city. The work was supported by the World Health Organization (18-TSA-005). The excellent technical assistance of Mervi Eskelinen, Päivi Hirttiö, Alena Kaijalainen, Elisa Lamminsalo, Marja-Liisa Ollonen, Eija Penttilä and Johanna Rintamäki are greatly appreciated.

*Erratum: The name of the fifth author in this article had been mis spelled. The mistake was corrected on 22 June 2010 and we apologise to the author.

\section{References}

1. Kew OM, Sutter RW, de Gourville EM, Dowdle WR, Pallansch MA. Vaccine-derived polioviruses and the endgame strategy for global polio eradication. Annu Rev Microbiol. 2005;59:587-635.

2. Minor P. Vaccine-derived poliovirus (VDPV): Impact on poliomyetlitis eradication. Vaccine. 2009;27(20):2649-52.

3. Centers for Disease Control and Prevention (CDC). Update on vaccine-derived polioviruses--worldwide, January 2008-June 2009. MMWR Morb Mortal Wkly Rep. 2009;58(36):1002-6.

4. Centers for Disease Control and Prevention (CDC).Update on vaccine-derived polioviruses. MMWR Morb Mortal Wkly Rep. 2006;55(40):1093-7.

5. World Health Organization (WHO). Guidelines for environmental surveillance of poliovirus circulation. World Health Organization, Geneva 2003.

6. World Health Organization (WHO). Polio Laboratory Manual. World Health Organization, Geneva 2004.

7. Blomqvist S, Savolainen C, Laine P, Hirttiö P, Lamminsalo E, Penttilä $E$, et al. Characterization of a highly evolved vaccinederived poliovirus type 3 isolated from sewage in Estonia. J Virol. 2004;78(9):4876-83.

8. Horie H, Koike S, Kurata T, Sato-Yoshida Y, Ise I, Ota Y, et al. Transgenic mice carrying the human poliovirus receptor: new animal models for study of poliovirus neurovirulence. J Virol. 1994;68:681-8.

9. Laboratory surveillance for wild and vaccine-derived polioviruses, January 2007-June 2008. Wkly Epidemiol Rec. 2008;83(36):321-8.

10. Rosen FS, Cooper MD, Wedgwood RJ. The primary immunodeficiencies. N Engl J Med. 1995;333(7):431-40. 